\title{
Radiation-induced Degradation of D-Glucose in Anaerobic Condition
}

\author{
Shunro KaWAKishi, Yukio Kito and Mitsuo Namiki \\ Department of Food Science and Technology, \\ Nagoya University, Nagoya, Japan
}

Received December 9, 1976

\begin{abstract}
Gamma-radiolosis of D-glucose in aqueous solution under anaerobic condition was investigated. D-Glucose was significantly decomposed by action of a hydroxyl radical, a primary radiolytic product of water. Under anaerobic condition, glucose radicals formed by hydrogen abstraction of hydroxyl radical were dehydrated to give deoxygenated sugars, and a part of the radicals followed disproportionation reaction to afford dicarbonyl sugars. Among the products formed through these reactions, 2-deoxy-D-arabinohexono-1,4-lactone ( $\mathrm{G}$ in $\mathrm{N}_{2}=0.6, \mathrm{G}$ in $\mathrm{N}_{2} \mathrm{O}=0.8$ ), 3-deoxy-D-erythrohexosulose and other 3-deoxy sugars as deoxygenated compounds, and D-arabinohexosulose and D-xylohexos-5-ulose as dicarbonyl compounds were identified. From these results, the radiolytic scheme of D-glucose in the absence of oxygen was proposed.
\end{abstract}

Previously, the authors reported that $\gamma$ radiolysis of $\mathrm{D}$-glucose in its air-free solution forms mainly 2-deoxy-D-arabinohexono-1,4lactone $^{1)}$ and also other deoxyhexosuloses and hexosuloses as minor products, ${ }^{2)}$ while in aerated solution, only dicarbonyl sugars are produced. $^{3)}$ On the other hand, it is well known that the irradiated glucose solutions exhibit bactericidal and bacteriostatic effects ${ }^{4,5}$ and also enhancement of browning reaction with amino acids, ${ }^{6,7)}$ and that the former is significantly enhanced in the presence of oxygen during irradiation. These facts indicate that the radiolysis processes of glucose are considerably different in the presence or absence of oxygen during irradiation.

Radiolytic degradations of glucose in their dilute aqueous solution are initiated primarily by action of hydroxyl radicals ${ }^{8)}$ and decomposed to many kinds of products including unidentified deoxygenated compounds. ${ }^{9 \sim 11\}}$ However, the products which have been identified by other workers were not necessarily the major components in the irradiated glucose solution and, in addition, it was not yet clear which kinds of products exhibit these effects mentioned above.

From these situations, we have reinvestigated in details the radiolytic process of glucose under anaerobic condition to make clear the differences in radiolytic mechanisms of glucose between aerobic and anaerobic conditions.

\section{EXPERIMENTAL METHOD}

Materials and irradiation. D-Glucose and all the other reagents used were of Guaranteed Grade. DGlucose solution $(20 \mathrm{~mm})$ prepared with triply distilled water was irradiated with $\gamma$-rays from a ${ }^{B 0} \mathrm{Co}$ source $(4 \mathrm{kCi})$ at a dose rate of $8.7 \times 10^{4} \mathrm{rad}$ per hour. Bubbling of purified nitrogen gas was performed prior to irradiation to remove oxygen in solution unless otherwise indicated.

Determination of glucose. The amount of residual glucose in the irradiated solution was determined by g.l.c.* method using D-sorbitol as an internal standard. Two milliliters of the irradiated solution and $3 \mathrm{mg}$ of $\mathrm{D}$-sorbitol in $0.5 \mathrm{ml}$ of water were mixed in a small test tube and freeze-dried. The dried matter was dissolved in $1 \mathrm{ml}$ of anhydrous pyridine and added with $0.4 \mathrm{ml}$ of hexamethyldisilazane and $0.2 \mathrm{ml}$ of trimethylchlorosilane, and the mixture was heated at $70^{\circ} \mathrm{C}$ for $10 \mathrm{~min}$. TMS** derivatives of the irradiated glucose were submitted to gas-chromatograph under the following conditions: Hitachi Gas Chromatograph model K 53, column; $3 \%$ SE-52 on Chromosorb W $0.3 \times 200 \mathrm{~cm}$, column temp. $180^{\circ} \mathrm{C}$, injection temp. $280^{\circ} \mathrm{C}$, carrier gas $\mathrm{N}_{2} 20 \mathrm{ml}$ per min. The amounts of residual glucose were calculated from the calibration curve.

* Gas-chromatographic. ** Trimethylsilyl. 
Determination of 2-deoxy-D-arabinohexono-1,4-lactone Essentially similar g.1.c. method was applied to the determination of this lactone and the authentic lactone prepared from the irradiated glucose ${ }^{1)}$ was used as a standard sample.

Preparation of alditol acetates from radiolytic products. The irradiated solution was treated with excess $\mathrm{NaBH}_{4}$ below $50^{\circ} \mathrm{C}$ to reduce all carbonyl compounds to their corresponding alcohols. After the reduction, Amberlite IR-120 $\left(\mathrm{H}^{+}\right)$resin was slowly added to the mixture until evolution of hydrogen gas ceased. The solution was filtered and evaporated under reduced pressure to dryness, and boric acid was removed from the residue by repeated distillation with methanol. The residue was acetylated with acetic anhydride containing $\mathrm{H}_{2} \mathrm{SO}_{4}(2.5 \%)$ at $60^{\circ} \mathrm{C}$ for $10 \mathrm{~min}$. For the preparation of deuterated alditol acetates, the freeze-dried matter was dissolved in $\mathrm{D}_{2} \mathrm{O}$, treated with $\mathrm{NaBD}_{4}$ and then acetylated as described above.

Separation of dicarbonyl sugars. The irradiated glucose solution was slowly passed through Dowex $1 \mathrm{X} 8\left(\mathrm{HSO}_{3}^{-}\right)$column $(1.8 \times 26 \mathrm{~cm})$ to remove unreacted glucose, other aldoses and aldonolactones, and adsorbed dicarbonyl sugars were eluted with $0.2 \mathrm{M}$ $\mathrm{NaCl}$ after washing the column with water. The eluate with $0.2 \mathrm{M} \mathrm{NaCl}$ was freeze-dried and desalted with methanol. Sephadex G-10 $(5 \times 68 \mathrm{~cm})$ and LH-20 $(2.2 \times 55 \mathrm{~cm})$ columns were used for complete desalting and separation of dicarbonyl sugars, with $0.01 \mathrm{M}$ phosphate buffer $(\mathrm{pH} 7.0)$ and $80 \%$ ethanol as eluting solvents, respectively.

Gas chromatography and mass spectrometry of alditol acetates. G.l.c. conditions: Hitachi Gas Chromatograph model $\mathrm{K} 53$; column, $8 \%$ silicone DC QF-1 on Chromosorb W (AW) $0.3 \times 200 \mathrm{~cm}$ stainless steel column, column temp. $220^{\circ} \mathrm{C}$, injection temp. $280^{\circ} \mathrm{C}$, carrier gas, $\mathrm{N}_{2} 20 \mathrm{ml} / \mathrm{min}$. Each alditol acetate preparatively collected from g.l.c. was submitted to mass spectrometry, using a JOEL-D-100.

\section{RESULTS}

1. Effects of oxygen and radical scavengers on the radiolysis of glucose

Since it is known that the first process of the radiolysis in dilute aqueous solution of glucose is initiated by action of radical species derived from water, the rate of decomposition of glucose should be influenced by the presence of oxygen or radical scavengers. Therefore, analyses for residual glucose in the solutions irradiated under different conditions were performed, i.e., (1) after bubbling with nitro-

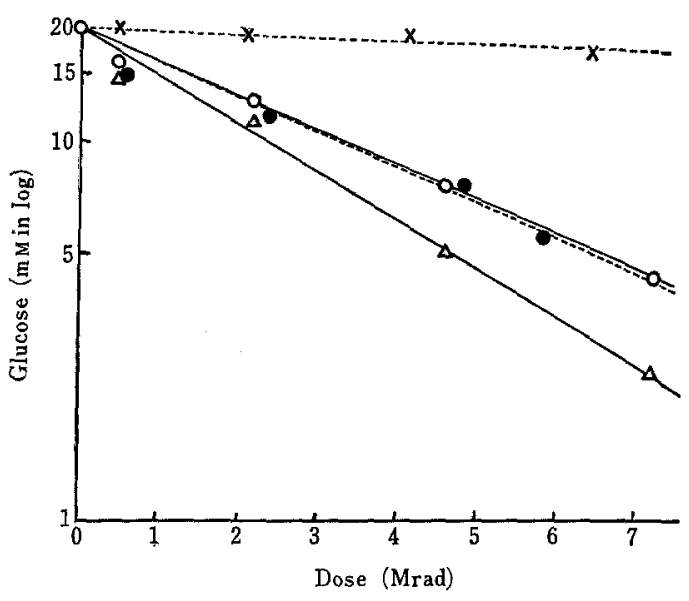

FIG. 1. Degradation of Glucose with $r$-Rays in Aqueous Solution.

Glucose $20 \mathrm{~mm}, \mathrm{O}-\mathrm{O}$, in $\mathrm{N}_{2} ;---\mathrm{O}$, in air; $\triangle-\Delta$, in $\mathrm{N}_{2} \mathrm{O} ; \times-\cdots \times$, in $\mathrm{KSCN}(40 \mathrm{~mm})$.

gen, (2) under continuous aeration, (3) with addition of KSCN ( $40 \mathrm{~mm}$ ) under deaerated condition, and (4) after saturation with $\mathrm{N}_{2} \mathrm{O}$. As shown in Fig. 1, the logarithmic decrease of the residual glucose with increasing radiation doses was linear in either case. While the decomposition rate was not affected by aeration, it was accelerated by the addition of $\mathrm{N}_{2} \mathrm{O}$. The presence of $\mathrm{KSCN}$, however, exhibited a complete protection of glucose from its reaction with a hydroxyl radical.

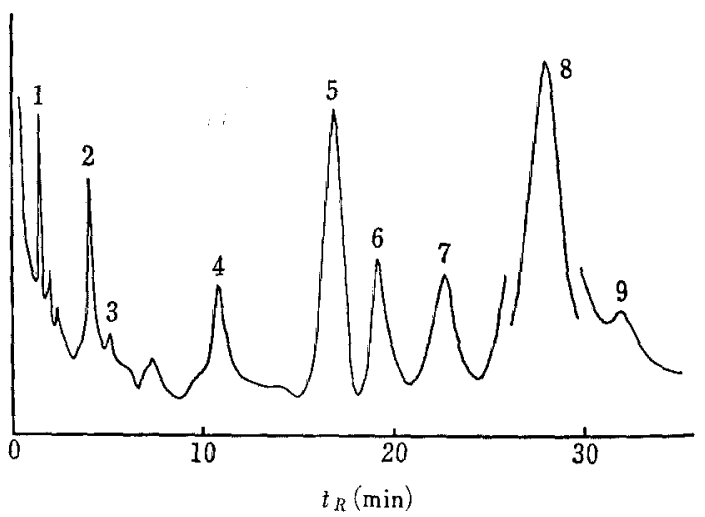

FIG. 2. G.1.c. of Alditol Acetates Prepared from Radiolytic Products of Glucose.

Column; $8 \%$ DC QF-1 on Chromosorb W $(0.3 \times$ $200 \mathrm{~cm}$ ), temp. $220^{\circ} \mathrm{C}$, inject temp. $280^{\circ} \mathrm{C}$, carrier gas $\mathrm{N}_{2} 20 \mathrm{ml} / \mathrm{min}$. 1, glycerol; 2, erythritol; 3, threitol; 4, methyl 2-deoxygluconate; 5, 2-deoxyglucitol; 6, 3-deoxyglucitol; 7, mannitol; 8, glucitol; 9, iditol. 


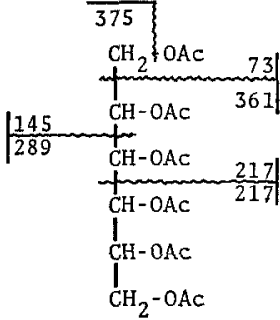

Hexitol Acetate

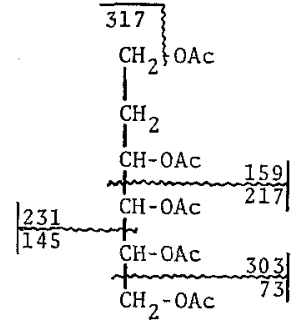

2-Deoxyhexitol Acetate

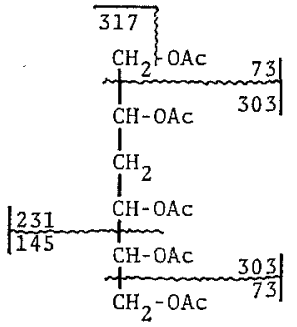

3-Deoxyhexitol Acetate

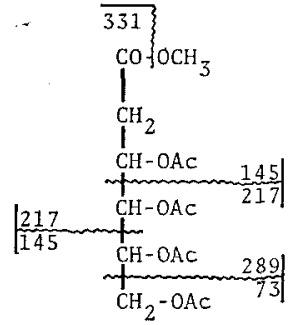

Methyl 2-Deoxyhexonate

FIG. 3. Mass Fragmentations of Several Hexitol Acetates.

\section{Identification of radiolytic products}

From $20 \mathrm{ml}$ of glucose solution $(20 \mathrm{~mm})$ irradiated with 2 Mrad under anaerobic condition, alditol acetates corresponding to the radiolytic products were prepared by the method described in a previous section. The gas chromatogram (Fig. 2) of the alditol acetates showed the formation of many decomposition products in the irradiated solution. Among these products, six alditol acetates (peaks 1, 2, 3, 7, 8 and 9) were identified by comparison of their relative retention times with those of the authentic samples. Peaks 4,5 and 6 were identified as respective acetates of methyl 2-deoxygluconate, 2-deoxyhexitol and 3-deoxyhexitol from their mass spectra. Mass fragmentations of these acetates are summarized in Fig. 3, in which are shown the specific fragment ions depending on their structures. There were a marked difference in the relative strength of the fragment ions, $m / e 159$ and 231, between 2-deoxyhexitol acetate (peak 5) and 3-deoxyhexitol acetate (peak 6), that is, 98 and $13 \%$ in the former, and 17 and $65 \%$ in the latter, respectively. Thus $m / e 159$ is taken as a specific fragment ion for 2-deoxyhexitol acetate and $m / e 231$ for 3-deoxyhexitol acetate. Peak 4, methyl 2deoxygluconate, was considered to be an artifact, because 2-deoxygluconic acid would be methylated during the treatment with excess methanol for the removal of boric acid produced by the decomposition of $\mathrm{NaBH}_{4}$, while 2-deoxygluconolactone was reduced to 2-deoxyglucitol (peak 5).

Since the dicarbonyl sugars are adsorbed on Dowex $1 \mathrm{X8}\left(\mathrm{HSO}_{3}^{-}\right)$column, it is possible

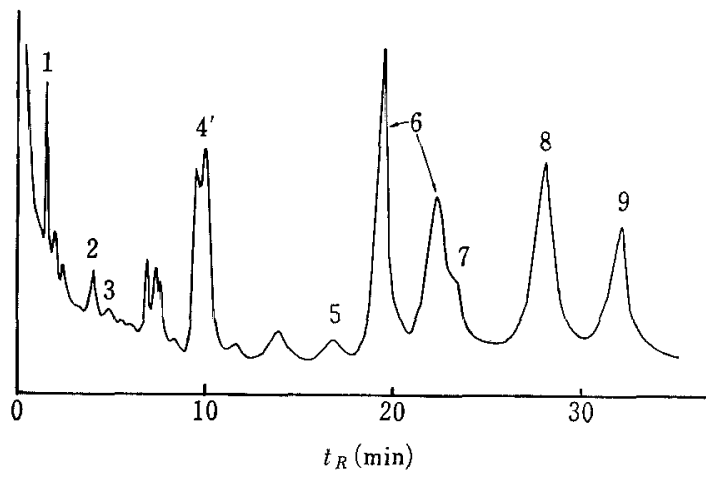

FIG. 4. G.1.c. of Alditol Acetates Prepared from Dicarbonyl Sugar Fraction of Irradiated Glucose.

G.1.c. condition and identification of each peak; details in Fig. 1. Peak 4'; mixture of ribitol and arabinitol acetates.

to separate them from unreacted glucose and other products. After passing the irradiated glucose solution slowly through Dowex column, dicarbonyl sugars were eluted with $0.2 \mathrm{M}$ $\mathrm{NaCl}$, followed by desalting and fractionation using Sephadex G-10 and LH-20 columns. The fraction containing dicarbonyl sugars was reduced, acetylated, and submitted to g.l.c. (Fig. 4). Each alditol acetate was identified from its relative retention time and its mass spectrum. Comparing Fig. 4 with Fig. 2, the disappearance of most of glucitol, 2deoxyhexitol and methyl 2-deoxyhexonate was observed. This means that most of glucitol in Fig. 2 is due to unreacted glucose, and that the latter two must have been derived from the products other than deoxydicarbonyl compounds. These alditols would be derived from various deoxyaldosuloses and aldosuloses produced by the radiolysis of glucose. To 
determine the position of carbonyl group newly formed in glucose molecule, the freezedried matter of the irradiated solution was treated with $\mathrm{NaBD}_{4}$ in $\mathrm{D}_{2} \mathrm{O}$ to form $>\mathrm{CD}$ OD from $>\mathrm{C}=\mathrm{O}$, acetylated, fractionated by preparative g.l.c., and subjected to mass spectrometry. The position of deuterium atom in the alditol-d acetate was determined by comparing its mass fragmentation with the corresponding alditol acetate. Mass spectrum of methyl 2-deoxygluconate acetate (peak 4) obtained by the treatment of $\mathrm{NaBD}_{4}$ was identical with that of $\mathrm{NaBH}_{4}$, and hence peak 4 was identified as methyl 2-deoxygluconate acetate. Peak 5 was shown to be 2 -deoxyglucitol- $\mathrm{d}_{2}$ acetate from the mass peaks at m/e $319\left(\mathrm{M}^{+}-\mathrm{OAc}\right), 305\left(\mathrm{M}^{+}-\mathrm{CH}_{2} \mathrm{OAc}\right)$ and $161\left(\mathrm{CD}_{2} \mathrm{OAc} \cdot \mathrm{CH}_{2} \cdot \mathrm{CHOAc}\right)^{+}$. Based on the fragment jons, $m / e 319$ and 289, it was estimated to be 2-deoxyglucitol-1,1- $\mathrm{d}_{2}$ acetate which would be derived from 2-deoxy-Darabinohexono-1,4-lactone (I). Mass spectrum of peak 6, 3-deoxyhexitol-d acetate, also indicated the presence of two deuterium atoms from the fragment ion $m / e 305$, and the ion pairs of $m / e 145 / 146,232 / 233$ and $304 / 305$ suggested the possibility that it was a mixture of four deoxyhexitol- $\mathrm{d}_{2}$, i.e., 3-deoxyhexitol1,2- $\mathrm{d}_{2},-1,4-\mathrm{d}_{2}, 4-\mathrm{deoxyhexitol}-1,3-\mathrm{d}_{2}$ and $-1,5-$ $\mathrm{d}_{2}$ acetate. These deoxyhexitols would be derived from 3-deoxy-D erythrohexosulose, 3deoxy-D-erythrohexos-4-ulose, 4-deoxy-D-erythrohexos-3-ulose and 4-deoxy-L-threohexos-5ulose, respectively. Considering the large intensities of $m / e 145$ and 233 to $m / e 146$ and 232 , and the presence of $m / e 217$ (CHOAc. $\left.\mathrm{CHOAc} \cdot \mathrm{CH}_{2} \mathrm{OAc}\right), \quad 3$-deoxy - hexitol-1, 2- $\mathrm{d}_{2}$ seems to be a main component in peak 6 . Therefore, it was estimated that 3-deoxy-Derythrohexosulose (TII) is a major product in four possible 3-deoxyhexosulose.

Nextly, peaks 7,8 and 9 in Fig. 2 were identified as mannitol, glucitol and iditol acetate, respectively, from their retention times, and most of glucitol acetate must have originated from unreacted glucose. Peak 7 and 9 were identified as mannitol-1,2- $\mathrm{d}_{2}$ and iditol-1,5- $\mathrm{d}_{2}$ acetate, respectively, based on their fragment ion pairs $m / e 145 / 147,217 / 219$ and $289 / 291$ for the former, and the fragment ions $m / e 146,218,290$ and ion pair $362 / 363$ for the latter. These $d_{2}$ compounds would come from D-arabino-hexosulose (II) and Dxylohexos-5-ulose (IV), respectively. G.1.c. of alditol acetates prepared from dicarbonyl sugar fraction also indicated the presence of ribitol and arabinitol (Fig. 4) which seemed to be originated from D-erythropentosulose.

\section{Formation of 2-deoxy-D-arabinohexono-1,4-} lactone in various irradiation conditions

Previously, the authors reported the isolation and identification of this lactone from the $\gamma$-irradiated glucose solution. ${ }^{1)}$ Peaks 4 and 5 in Fig. 2 also indicated the formation of this lactone, which is known to be the main radiolytic product of glucose under anaerobic condition from their peak areas. D-Glucose solutions $(20 \mathrm{~mm})$ were irradiated at several doses (1) under nitrogen saturated (in $\mathrm{N}_{2}$ ), (2) under $\mathrm{N}_{2} \mathrm{O}$ saturated (in $\mathrm{N}_{2} \mathrm{O}$ ) and (3) with an addition of $40 \mathrm{mM} \mathrm{KSCN}$ (in KSCN), and the formed lactone detected as TMS ether was determined quantitatively by g.l.c. method. As shown in Fig. 5, the formation of the lactone was enhanced by $\mathrm{N}_{2} \mathrm{O}$ saturated, but depressed by addition of KSCN. Initial $G$ values for the lactone formation in $\mathrm{N}_{2} \mathrm{O}$ and in $\mathrm{N}_{2}$ were 0.8 and 0.6 , respectively. $\mathrm{N}_{2} \mathrm{O}$

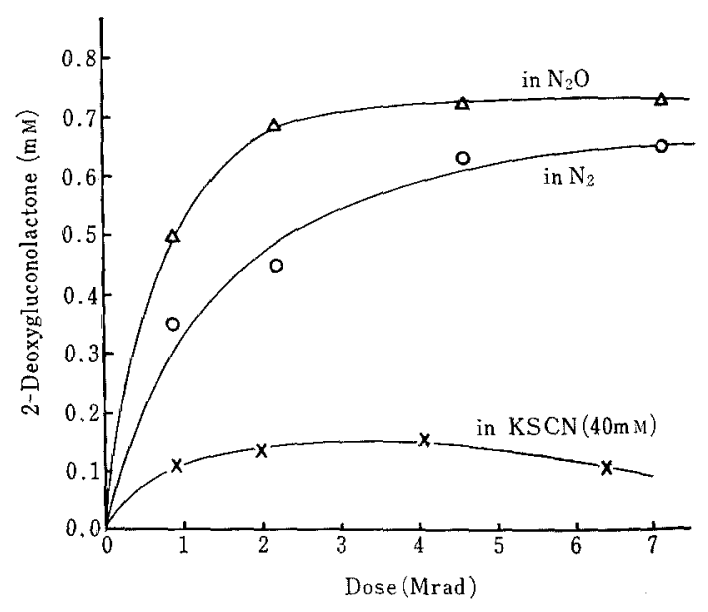

FIG. 5. Formation of 2-Deoxy-D-arabinohexono1,4-lactone in Irradiated Glucose Solution. 
is an effective scavenger for hydrated electron as $\mathrm{N}_{2} \mathrm{O}+\mathrm{e}_{\mathrm{aq}}^{-} \underset{\mathrm{H}_{2} \mathrm{O}}{\longrightarrow} \cdot \mathrm{OH}+\mathrm{OH}^{-}+\mathrm{N}_{2}$ and $\mathrm{KSCN}$ scavenges hydroxyl radical. Accordingly, it seems that 2-deoxy-D-arabino-hexono-1,4- lactone was formed by action of hydroxyl radical similar to the case of decomposition of glucose. This lactone presumably exists in equilibrium with its free acid in the irradiated solution. If $\mathrm{pH}$ of the solutions to be irradiated is varied from acidic to basic, the changes should be observed in this equilibrium to increase the acidic form and to decrease the lactone form, which are shown as an increase of peak 4 and a decrease of peak 5 in the g.l.c. (Fig. 2). The glucose solutions $(20 \mathrm{~mm})$ prepared with $\mathrm{M} / 15$ phosphate buffer were adjusted to $\mathrm{pH}$ $2.0,6.0$ and 10.0 using $1 \mathrm{~N} \mathrm{H}_{2} \mathrm{SO}_{4}$ or $1 \mathrm{~N}$ $\mathrm{NaOH}$, saturated with $\mathrm{N}_{2} \mathrm{O}$ and irradiated with $2 \mathrm{Mrad}$. After these solutions were reduced with $\mathrm{NaBH}_{4}$ and acetylated, alditol acetates were submitted to g.l.c. The gas chromatograms are shown in Fig. 6. A marked

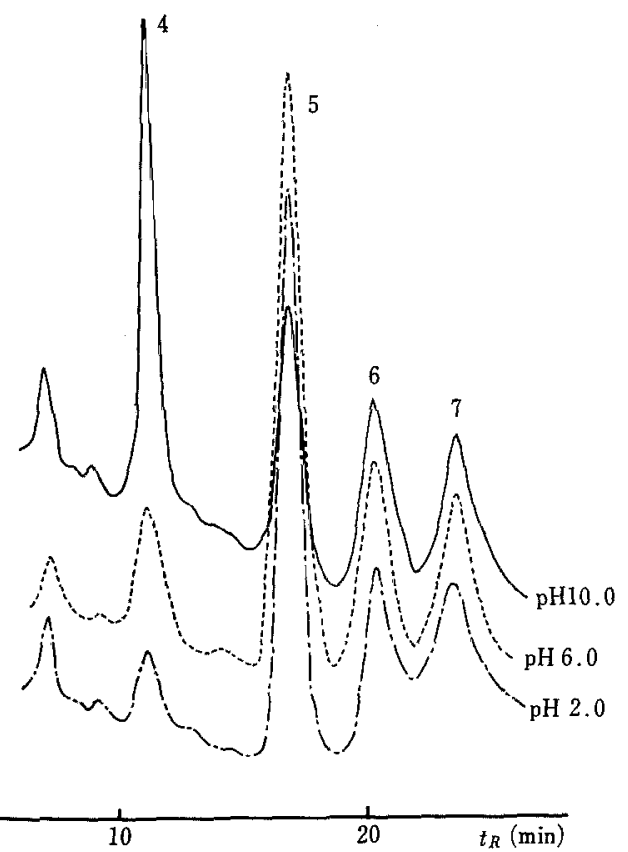

FIG. 6. Changes of Peak 4 and 5 in G.1.c. of Alditol Acetates Prepared from Glucose Solution Irradiated at Different $\mathrm{pH}$.

G.1.c. condition and identification of each peak; details in Fig. 1. increase of peak 4 (methyl 2-deoxy-gluconate acetate) and decrease of peak 5 (2-deoxy glucitol acetate) at $\mathrm{pH} 10.0$ were observed. This means that these two compounds are derived from 2-deoxy-arabinohexono-1,4-lactone.

\section{DISCUSSION}

D-Glucose is significantly decomposed by $\gamma$-radiolysis in aqueous solution, and its degradation is accelerated under the atomosphere of $\mathrm{N}_{2} \mathrm{O}$ which is a scavenger for hydrated electron, and effectively inhibited by addition of $\mathrm{KSCN}$ as a hydroxyl radical scavenger (Fig. 1). These facts suggest that the radiolysis of glucose is mainly proceeded by action of hydroxyl radical, a primary radiolysis product of water, as reported by Phillips et al. ${ }^{81}$ The radiolysis of glucose under aerobic and anaerobic conditions proceeded nearly at the same rates, but there were large differences between the radiolytic products and their formation mechanism under these conditions. These differences in the products are shown in Table $\mathrm{I}$, which contains only hexose derivatives in

Table I. Hexose Derivatives in Radiolytic Products of Glucose in N, AND In AIr Conditions

\begin{tabular}{|c|c|c|}
\hline Products & $\operatorname{in} \mathrm{N}_{2}$ & in $\mathrm{Air}^{33}$ \\
\hline \multicolumn{3}{|l|}{ 2-Deoxy-D-arabinohexono- } \\
\hline 1,4-1actone (I) & $+t+t$ & - \\
\hline 3-Deoxy-D-erythrohexosulose (III) & ++ & - \\
\hline D-Arabinohexosulose (II) & ++ & $++t$ \\
\hline D-Ribohexos-3-ulose & - & ++ \\
\hline D-Gluconolactone & \pm & $+t$ \\
\hline $\mathrm{D}-X y$ lohexos-4-ulose & - & + \\
\hline $\mathrm{D}$-Xylohexos-5-ulose (IV) & + & + \\
\hline
\end{tabular}

many radiolytic products of glucose. In a previous paper $^{3)}$ dealing with the radiolysis of glucose under aerated condition, the authors reported that several dicarbonyl hexoses were determined as main radiolytic products and deoxydicarbonyl hexoses were unable to be detected. These facts indicated that dicarbonyl hexoses are produced by the decomposition of glucose hydroperoxy radicals formed through 
the reaction of glucose radicals and oxygen. Under air-free condition, however, several deoxysugars were formed as main while dicarbonyl sugars were minor products, in contrast to the products formed under aerated condition.

Moreover, the deoxy and carbonyl groups newly formed in a glucose molecule were necessarily present in the adjacent positions. These findings lead us to assume that the following dehydration reaction may occur in glucose radical:<smiles>CC(C)C(=O)C=CC(C)C(C)O</smiles>

This dehydration seemed to be the same reaction with the radiolytic formation of acetaldehyde from ethylene glycol. ${ }^{12)}$ Dicarbonyl sugars as minor products may be processed by disproportionation of glucose radicals:

2

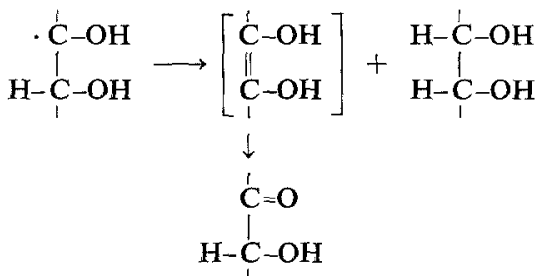

However, when air is supplied to the glucose solution during irradiation, a glucose radical quickly reacts with oxygen molecule to give glucose hydroperoxy radical, which is decomposed to dicarbonyl sugar and other bond-fission products. ${ }^{3}$ )

Therefore, the radiolysis mechanism of glucose under anaerobic condition can be assumed to be as follows: Glucose $\mathrm{C}-1$ radical 1 formed by hydrogen abstraction from glucose with hydroxyl radical is dehydrated to give the radical $\mathbf{2}$ which abstracts a hydrogen from other glucose molecule or reacts with hydrogen radical, the other radiolytic product of water, to produce 2-deoxygluconolactone (I). The $\mathrm{C}-2$ radical $\mathbf{3}$ gives 3-deoxy-Derythrohexosulose (III) via the radical 4 by dehydration and hydrogen abstraction as in the case of the $\mathrm{C}-1$ radical. Both radicals 1 and $\mathbf{3}$ are also oxidized to D-arabinohexosulose

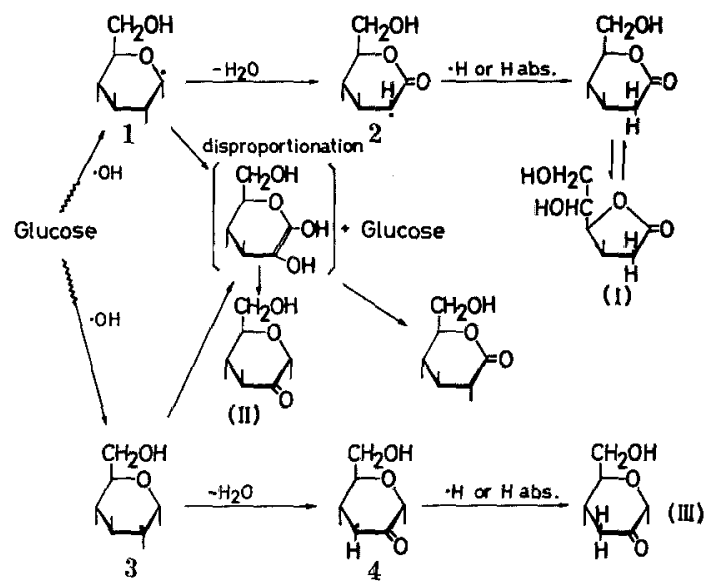

FIG. 7. Proposed Mechanism for Radiolysis of Glucose under Anaerobic Condition.

(II) by disproportionation, through 1,2-unsaturated glucose as an intermediate. A part of this intermediate should be also transformed to gluconolactone simultaneously with formation of (II), although only a small amount of lactone was detected in the present study. In these reactions, the formation of (I) and (II) seemed to be the main process of glucose radiolysis as evidenced by their high yields as shown in g.l.c. (Fig. 2). These processes are summarized in the following scheme (Fig. 7). Product (IV), D-xylohexos-5-ulose, may be formed from the $\mathrm{C}-5$ radical by disproportionation.

Moreover, one of the other characteristics on the radiolysis under air-free condition was a little of the scission products than the case under aerated condition, in which many scission products as triose, tetrose and pentose derivatives owing to the cleavage of carbon chain were identified. ${ }^{3 \text { i }}$

Finally, the difference in the radiolysis between glucose and fructose ${ }^{2}$ may be closely related to that any kinds of hexose radicals will be preferentially produced by hydrogen abstraction with hydroxy radical, that is, which of hydrogen atoms in hexose molecule will be easily abstracted. Main products from glucose and fructose on their radiolysis under air-free condition are (I) and 1-deoxy-Dthreo-2,5-hexodiulose, ${ }^{2,18,14)}$ which are derived 
from glucose $\mathrm{C}-1$ and fructose $\mathrm{C}-5$ radicals, respectively. Conclusively, it is suggested that the hydrogen at the $\mathrm{C}-1$ of glucose and the C-5 of fructose may be abstracted faster than the other hydrogens with hydroxyl radical.

\section{REFERENCES}

1) S. Kawakishi and M. Namiki, Carbohyd. Res., 26, 252 (1973).

2) S. Kawakishi, Y. Kito and M. Namiki, ibid., 30, 220 (1973).

3) S. Kawakishi, Y. Kito and M. Namiki, ibid., 39, 263 (1975).

4) J. Schubert and E. B. Sanders, Nature, New Biol., 233, 199 (1971).

5) M. Namiki, Y. Watanabe, J. Okumura and S.
Kawakishi, Agric. Biol. Chem, 37, 989 (1973).

6) S. Kawakishi, J. Okumura and M. Namiki, Nippon Nôgeikagaku Kaishi, 46, 459 (1972).

7) S. Kawakishi, Y. Kito and M. Namiki, IVth International Congress of Food Science and Technology, Madrid, Sept. 27, 1974.

8) G. O. Phillips, W. Griffiths and J. V. Davies, $J$. Chem. Soc. B, 1966, 194.

9) G. O. Phillips, G. J. Moody and G. M. Mattok, ibid., 1958, 3522.

10) P. M. Grant and R. B. Ward, ibid., 1959, 2871.

11) H. Scherz, Rad. Res., 43, 12 (1970).

12) F. Seidler und C. v. Sonntag, Z. Naturforsch., 24b, 780 (1969).

13) S. Kawakishi, Y. Kito and M. Namiki, Agric. Biol. Chem., 39, 1897 (1975).

14) M. Dizdaroglu, J. Leitich and C. v. Sonntag, Carbohyd. Res., 47, 15 (1976). 